\title{
AN INNOVATIVE SOCIAL MOBILE PLATFORM TO SUPPORT REAL-TIME COMMUNICATION IN PEER TUTORING
}

\author{
Meghan Wang ${ }^{1}$ and Yu Sun ${ }^{2}$ \\ ${ }^{1}$ Valencia High School, Placentia, USA \\ ${ }^{2}$ California State Polytechnic University, Pomona, USA
}

\begin{abstract}
This paper looks into the reasoning, context, and process behind the creation of the Step Up App to be used in the Step Up Club. The Step Up Club is a peer tutoring high school organization that allows students to tutor one another. The paper explains the background and the issues that exist with peer tutoring regarding challenges in communication between tutors and tutees. The app aims to provide the solution for many of those problems in creating a new platform in which students can communicate to one another about any questions regarding school academics.
\end{abstract}

\section{KEYWORDS}

Computer Science, Communication, Peer Tutoring App, Academic Help, Mobile Computing

\section{INTRODUCTION}

Peer tutoring is a method in which people who hope to learn help each other by teaching one another [1]. This less common form of tutoring could bring many advantages to learners and is being studied to see the advantages it creates [2]. In fact, the area of peer tutoring is being researched from all areas including not only educational but also social and psychological facets [3]. In recent years, high schools secondary education has also been a large area of discussion in investigating different areas of schools as well as changing existing curriculums to analyse students' performance [4]. Peer tutoring within high school students has already shown positive results [5].

This research paper explains how the Step Up App was created in order to help in peer tutoring communication that was absent in the organization before. The Step Up App is a peer tutoring App allowing students to message peers about questions concerning school or homework. It consists of a real-time chat, allowing students to join a large discussion room, or to message tutors on a one to one basis. The app also contains a leader board, from students ratings of tutors, allowing other students to evaluate how helpful certain tutors are.

High school students often face much stress and need help on their school work. School clubs and organizations as well as the Parent Teacher Association have all made efforts to provide tutoring or help in standardized testing for students. However, students often are unwilling or unable to afford tutoring and also may not feel that tutors who have not experienced the actual course can understand their problems.

Natarajan Meghanathan et al. (Eds) : ICCSEA, WiMoA, SPPR, GridCom, CSIA - 2017 
The Step Up Club is a high school organization at Valencia High School, created to help students receive one on one peer tutoring form other high school students for free. The idea of peer tutoring was implemented in order to allow students to receive help from other students who have had first hand experience in taking a class and mastering it or excelling in the course. Tutors for each of the subjects are chosen after submitting their grades in the course they have already completed.

However, the organization only provided help when students and tutors met up with one another in person. If students realized they needed help once they left the school's library where they would meet with tutors, it would be difficult to receive the help they needed. Other circumstances such as scheduling to meet with tutors in person also create similar challenges with a lack of other forms of communication.

The Step Up App creates a platform to solve this issue by allowing students to immediately communicate with tutors even at home, using the real-time chat. The leader board, displaying top ranked tutors are recognized and awarded by the club, providing an incentive for students to help others. The app allows a more convenient form of communication for both tutors and tutees.

This paper is organized as follows. Section 2 discusses the challenges that the Step Up organization faced which eventually led to the creation of the app. Section 3 discusses the solution the app has to offer to the challenges. Section 4 approaches how each solution works to solve the challenges. Section 5 discusses some other similar ideas to the Step Up App, and Section 6 concludes the research paper.

\section{Challenges}

The examples with many students, both tutors and tutees, led to a closer analysis at a pattern seen throughout of challenges the Step Up organization faced.

\subsection{Inconveniency}

One of the many challenges faced by the Step Up organization was the inconveniency of requiring tutors to meet face to face with tutees. As high school students, both parties face many tight schedules from after school competitions such as decathlon to competing in seasonal sports such as volleyball. Having to schedule sessions with peers became a monotonous process as both sides would repeatedly have conflictions in timing. This was one of the many inconveniences of the structure of the organization.

Additionally, having tutors and tutees meeting at school had an even narrower time frame due to the opening and closing hours of the high school's library, where students could meet and have textbooks available to borrow. This time frame lasted from when school ended at 2:45 to 4:00. The time frame is not only short, but leaves students very little amount of time in being able to schedule a tutoring session. Theses inconveniences led to much frustration with the students and an unwillingness to schedule sessions. Many students who try to meet with one another outside of school often have difficulties as well in attempting to find a proper location where both sides are easily able to meet at the same time.

\subsection{Lack of Motivation}

From the beginning of when the Step Up organization to present time, there has been a great trend in decreasing numbers of both tutors and tutees participating as a member of the club. When members first joined, there was a large group in both sides of the club, as this innovative organization was formed at the school. Students had higher participation rates as well as interest 
in the program. As the high school students began to move up in grade level, taking on more challenging courses and extracurricular activities, a fall in interest began showing due to the inconvenience in having to stay for complete sessions and scheduling with tutors.

Over time as the Step Up organization grew older, students began developing a lack of motivation in participation as both a tutor and tutee. A portion of the growth in lack of motivation throughout the club was due to the frustration and tenuous process of attempting to communicate between both parties in scheduling tutoring session. Throughout the year, tutoring sessions would diminish as students no longer wanted to take time out to schedule a session for simple questions concerning school work.

Furthermore, another contribution to the lack of motivation seen in participants of the Step Up organization was largely due to the fact of the creation of a competing organization at Valencia High School known as "Tiger Tutoring," that branched off of a larger organization known as National Honor Society. National Honor Society, or NHS, is a much larger nationwide organization for high schools that provides an incentive of recognition after student's participation in tutoring other students. NHS tutors would receive recognition through honor cords and a cap tassel for graduation. This newly created organization not only drew away participants from Step Up, but also created a new competition of tutoring to Step Up. It provided similar hours of community service hours to students and also brought in more incentives of being recognized for participating.

\subsection{Timing}

Issues concerning timing became a large challenge generally for tutors. One of the main issues with Step Up's original structure is its rigid form of communication and restricted person to person interactions. The inconveniency with scheduling sessions is not the only issue. A larger issue holds in that tutees often realize they need the most help in certain facets upon returning home and working on problems on their own. At these moments when tutees on their own, they need the help of tutors but are unable to because the tutors are only available through actual meetings.

Scheduled tutoring sessions also incur timing issues with certain deadlines that teachers have set for students. Because of the packed schedules every student takes on, there may be occurrences where tutors and tutees can not decide on a meeting time to meet in person before the deadline of a large project or a test date.

\subsection{Challenge faced by Specific Student}

In the year 2015-2016, one female student facing difficulties in understanding the content of her chemistry course was recommended by her teacher in receiving help from a tutor at Step Up Club. Over the semester, the tutor and tutee would meet up after school, in the library at school to receive help. However, the tutee mentioned numerous times that she often struggled with her work at home even after receiving help at school. The tutee had many questions that she had not thought of to ask the tutor until returning home and reviewing the topics of the unit.

\section{SOLUTION}

The problem surrounding many of the challenges with the original Step Up organization lies in the rigid structure of the club. Through the app itself along with a leader board and real-time chat room for discussion sessions, the Step Up App is created as the solution to many original problems. 
Figure 1 shows an overview of the architecture of the app. The app is based on a typical serverclient communication model. We have implemented the app in Android, while the backend is supported by Google Firebase. The reason to choose Firebase is its enhanced support on real-time data communication and synchronization.

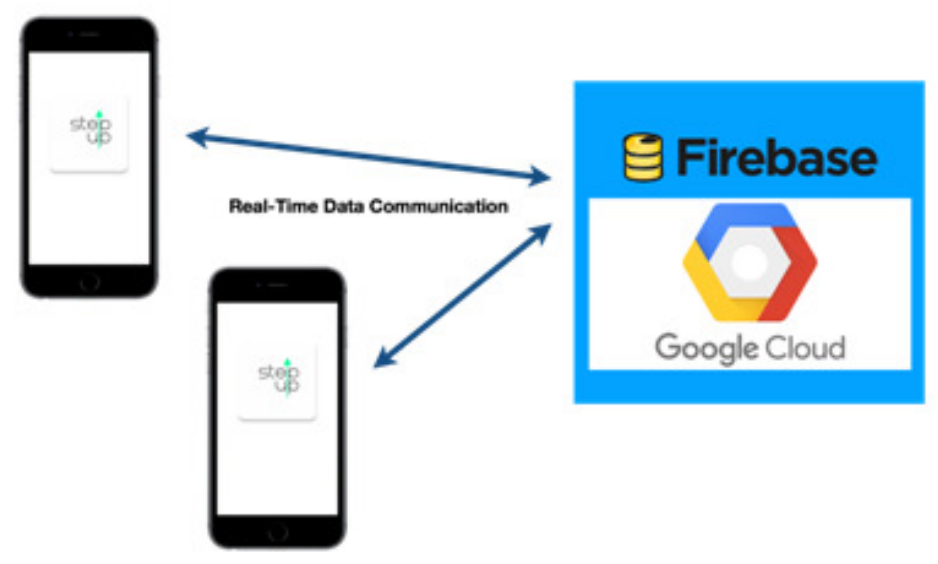

Figure 1. The Architecture of the Step Up App

\begin{tabular}{|c|c|c|c|c|c|c|c|}
\hline \multicolumn{8}{|c|}{$\begin{array}{l}\text { US Smartphone User Penetration, by Age, 2014-2020 } \\
\text { \% of mobile phone users in each group }\end{array}$} \\
\hline & 2014 & 2015 & 2016 & 2017 & 2018 & 2019 & 2020 \\
\hline $0-11$ & $28.7 \%$ & $35.3 \%$ & $41.0 \%$ & $45.0 \%$ & $47.1 \%$ & $48.9 \%$ & $49.7 \%$ \\
\hline $12-17$ & $71.0 \%$ & $78.5 \%$ & $84.0 \%$ & $89.0 \%$ & $91.0 \%$ & $92.0 \%$ & $92.9 \%$ \\
\hline $18-24$ & $85.2 \%$ & $90.1 \%$ & $94.9 \%$ & 98.096 & $98.4 \%$ & $99.0 \%$ & $99.4 \%$ \\
\hline $25-34$ & $84.0 \%$ & $90.2 \%$ & $95.2 \%$ & $97.0 \%$ & $97.1 \%$ & $97.2 \%$ & $97.3 \%$ \\
\hline $35-44$ & $81.6 \%$ & $87.7 \%$ & $92.0 \%$ & $94.0 \%$ & $94.8 \%$ & $96.1 \%$ & $97.0 \%$ \\
\hline $45-54$ & $66.9 \%$ & $75.8 \%$ & $82.8 \%$ & $88.3 \%$ & $92.8 \%$ & $95.9 \%$ & $97.9 \%$ \\
\hline $55-64$ & $59.1 \%$ & $67.6 \%$ & $75.7 \%$ & $80.9 \%$ & $85.5 \%$ & $89.5 \%$ & $93.0 \%$ \\
\hline $65+$ & $36.6 \%$ & $40.7 \%$ & $44.4 \%$ & $49.6 \%$ & $52.8 \%$ & $55.7 \%$ & $55.4 \%$ \\
\hline Total & $67.6 \%$ & $73.8 \%$ & $79.0 \%$ & $82.7 \%$ & $84.8 \%$ & $\mathbf{8 6 . 5} \%$ & $87.3 \%$ \\
\hline \multicolumn{8}{|c|}{$\begin{array}{l}\text { Note: individuals who own at least one smartphone and use the } \\
\text { smartphone(s) at least once per month } \\
\text { Source: eMarketer, Feb } 2016\end{array}$} \\
\hline
\end{tabular}

Figure 2. eMarketer's Table of US Smartphone User Penetration [6]

\subsection{Holistic App}

The design of the app itself has the main goal of portability and ease of usage. The original issue was due to the inflexible composition of the club in having only person to person real life meetings. The app offers a real-time chat that allows tutors and tutees to maintain and create tutoring sessions even outside scheduled meetings.

The rationale behind the creation of the app was to create a system that could be used anywhere and anytime, to increase an efficiency and convenience for all members of the club. The idea of an app as the larger goal to attack the challenges emerged from noticing the everyday usage of people on apps. Almost all high school students have smart phones that they carry around on a daily basis. Creating an app that can be easily downloaded on these hand held devices becomes a much easier process for all students rather than having to schedule and meet someone in person. eMarketer has in fact studied teenagers usage of smart devices as seen in the chart below, and the 
trend has only shown an increase in the percentage of the teenage age group [6]. The trend even extends to 2020. Implementing an educational app for teenagers to use may also add a positive impact to the large usage of young people in mobile devices.

\subsection{Leader Board}

The leader board is a feature in the app that consists of a rating system for students on each tutor. The rationale behind creating such a screen within the app was to offer an incentive to students. As seen in the competition of the tutoring organization known as Tiger Tutoring created by the National Honor Society which offered a recognition of being a tutor, the system of a Leader Board similarly offers a recognition. In fact the leader board offers a more competitive form of recognition because the ratings between every tutor becomes an incentive that makes members of Step Up all strive to become better tutors for one another.

The rationale behind the leader board was not only to create this form of motivation but also in bringing in an element of reliability for students. In having such a rating system, students become more aware and are able to differentiate from the better and worse tutors. This creates a much more trustworthy system that provides the best form of resources for the students who are tutees needing help in a particular area.

\subsection{Real-Time Chat}

The main feature and idea behind creating the Step Up App was to implement a real-time chat that allows for the flexibility in time between tutors and tutees. The rationale behind the app was its ease in being used between tutors outside of scheduled tutoring sessions. It not only provides a connection between original tutors and tutees that continue meeting both in person and through messaging, but it may also induce new participants because of its flexibility and lack of binding between a tutor and tutee. Additionally, the rationale behind the real-time chat between two people also led to a larger group discussion that included all members. The idea behind the larger group discussion was to promote a flexibility in timing of allowing all people to answer in urgent need. However, the basic idea of one to one tutoring that serves as the foundation of the Step Up club is still maintained.

The real-time chat is implemented using the data service - Firebase [7] hosted in Google Cloud [8]. Firebase is a popular mobile backend as a service solution to support real-time data synchronization and communication across multiple clients. The messages that are sent within the chats on the app are all stored through the online Firebase database. Individual chat rooms between a tutor and tutee is stored under the tag "Tutor Messages," stored by time order as value under the tag of the user's name. The larger chat room with all users is identified under the tag of "Messages" and the values are in order the time in which the messages were sent as well.

\section{METHODOLOGY OF SOLUTION/EMPIRICAL RESULTS}

The Step Up App is a new edition to the Step Up organization that provides numerous elements and methods in helping solve many of the issues faced including inconveniency, lack of motivation, along with timing. The app also aims to create a new feature to the organization as a whole. The methodology in this section serves to explain in detail each of the specific features of the app that serve as solutions to particular challenges.

\subsection{Solving Inconveniency}

The Step Up App helps in solving many of the issues surrounding the inconveniency of the rigid original structure of the organization. The app itself is the main solution to the inconveniency 
through its portable method of communication between tutors and tutees. With the publication of the app, the two parties no longer have to schedule person to person meetings and rather have a new means to communicate with each other whenever and wherever. The real-time chat allows for conveniency between tutors and tutees in much more efficiently being able to communicate with one another regardless of each sides' schedules.

In the situation where tutors and tutees schedules conflict, the tutee could easily first ask the tutor a question using the app. Once the tutor has the time to respond, they could quickly respond to one another. Students could also use the app to potentially schedule a real life meeting in person with their tutors if they hope to do so. The app provides a new platform that still permits the original basic composition and purpose of the Step Up organization, but adds along a new highlight to the club. Students who still prefer the original person to person contact still have the more intimate method available. At the same time, if needed, they have the app to remain in contact with tutors at home. During busier testing seasons, both tutors and tutees have a much more convenient method of communication through the app. Students who face trouble in finding methods to meet up outside of school similarly can use the app as a form of communication.

The app itself is also incredibly easy to move from screen to screen, whether signing in, creating a new account, or chatting with tutors. People can easily install the app on their phones from the Play Store, create and account, and begin interacting with tutors. Each of the features of the app can be selected from the "Home Page" that users immediately see upon signing into the app. When students select particular tutors, they are able to chat with them one on one. Otherwise, students can also join the large chat room that hold all registered members of Step Up and have discussions of their topic.

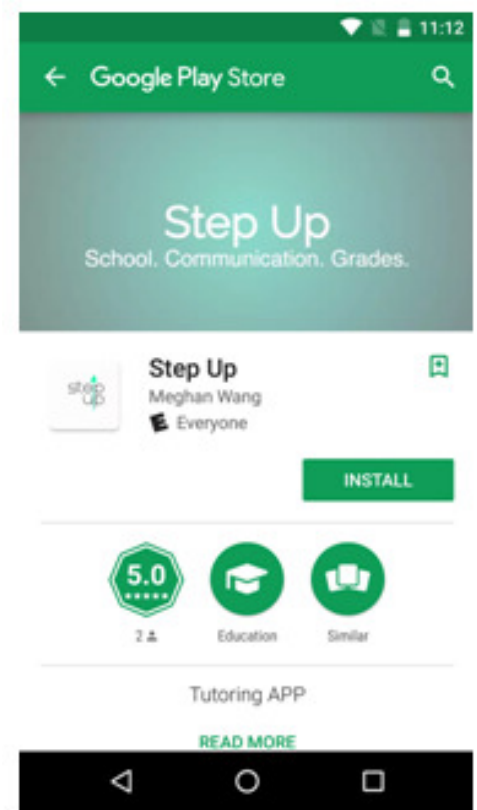

Figure 3. Screenshot From Smart Phone of Google Play Store

\subsection{Creating Motivation}

Another large issue the Step Up organization faced was in the lack of motivation from both tutors and tutees over time. The majority of this issue is solved with the publication of the app yet again. A decrease in motivation was largely seen due to the lack of conveniency that previously existed 
when tutors and tutees had to go through long processes of scheduling with one another repeatedly and each facing differences in timing that eventually led to frustration and irritation, causing the lack of motivation. The app helps in solving the issue of the inconveniency.

The leader board portion of the app is also an important component that helps in creating the motivation necessary for the success of the organization. The leader board element of the Step Up App is a screen that ranks the top ten best tutors, as seen ranked by other students who have received help from those tutors. Students are able to rank each tutor on their profile pictures with a rating of five being "extremely helpful" to one being "not very helpful." The leader boards uses a function made during the creation that averages the rankings of each tutor and lists them out. At the conclusion of each month, the top ranked in the leader will be recognized for their help and dedication to the organization.

One of the other factors to the fall of motivation in members of the Step Up club can mainly be attributed to the existence of the competition from the other organization of tutoring created at the school by the National Honor Society. The addition of the Step Up App helps diminish much of the lack of motivation in participating in Step Up because of the new feature that has been created that the competition lacks. It creates a new highlight that will draw members into because of its innovative aspect.
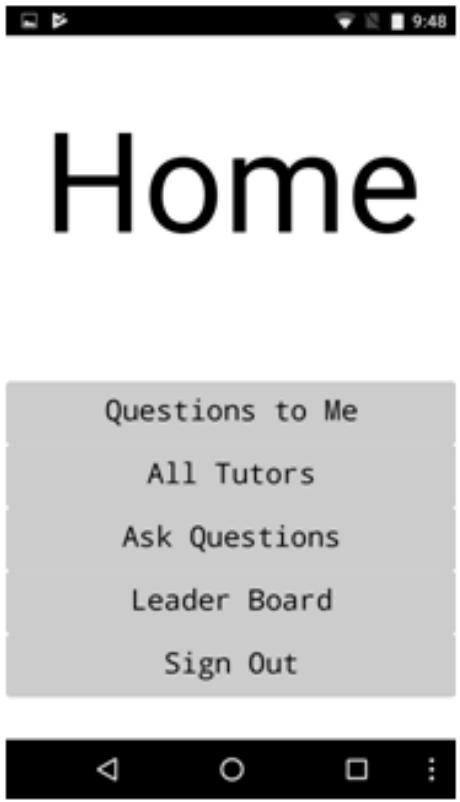

Figure 4. Home Page of Step Up App

\subsection{Establishing Much More Flexible Timing}

Timing becomes much more flexible and no longer is a challenge because of the Step Up App. Using the app, tutors and tutees can easily communicate with one another from anywhere at anytime. The rigid scheduling issue is solved with this flexibility. Students having various schedules no longer need to face the original issue. The challenge surrounding timing of how students having questions after tutoring sessions is thus also solved. When tutees are at home, working and realize there is information they do not understand and did not originally imagine it as a difficulty, can easily contact their tutor with ease through the app. 
Additionally, if students face challenges on their own and ask their tutor, but the tutor missed the message, forgot to respond, or is busy, the issue with tight deadlines may seem to arise again. However, students are always able to join the large chat room and discuss with all other students or they have the ability to contact all other tutors one-to-one by selecting their profile and discussing if they prefer to do so in that manner. The flexible timing with the app helps resolve issues against the less flexible timing with school assignments, tests, and large projects by offering help and communication that is essentially unrestricted by timing.

\section{RELATED WORK}

The Step Up App provides a Peer-to-Peer Tutoring that some other Apps and online resources also contain similarities too and also offer similar services.

\subsection{Sesh Mobile App}

The Sesh mobile app was created by a company from Vanderbilt University and Stanford University students, known as Vanford, and the purpose was to allow students to request for tutoring sessions during any time. The company wanted to create a more expansive community of learning for students leading to the creation of the app and it started with only the two universities but hopes to expand. The app was used to request in-person sessions, also known as a "sesh" with tutors who have had to apply by uploading their transcripts of what classes they offer. Each tutor earns $\$ 20$ per hour [9].

This app is similar to the Step Up App in the hopes of creating a community where all students are able to communicate about academics in one area. However, the Sesh mobile app is used solely to request an in-person tutoring meeting. The goal of the Step Up App is to allow both inperson as well as easily accessible online communication to create efficiency for students. Additionally, Step Up has no requirement for tutors to submit an application because all members of Step Up are permitted to help others. While an application can assure reliability, the Step Up App is made reliable through a tutee rating system. Additionally, Step Up has no costs at all to receive the help students need. The Sesh App promotes an all day and night availability, but this is much more easily achieved through Step Up which does not require for either tutors or tutees to travel when the tutee needs help late at night.

\subsection{Brainfuse}

The Brainfuse online tutoring website offers help to students through a 24/7 online tutoring and writing lab system. It offers an online peer tutoring system that allows students to contact one another as they believe in peer tutoring as the method that often allows students to learn more easily. The "Online Learning Platform" of Brainfuse is aimed at helping colleges and universities in creating a peer-to-peer learning community [10].

The Step Up App has many similarities to Brainfuse in its core idea of a providing a community for students to peer tutor one another. While the Brainfuse website also provides an actual applied tutor as well, it is only focused on colleges and universities. The Step Up App is designed as a part of an organization in one high school, but aims at being user friendly to people in all levels of education. Additionally, the mobile App itself is effortlessly accessible with a click away on the phone that an online website does not provide for. 


\section{CONCLUSION AND FUTURE WORK}

The Step Up App is an innovative approach in bringing in a peer tutoring service to a larger community. It provides a new feature to the Step Up organization in peer tutoring by solving the original challenges faced including inconvenience, lack of motivation, as well as inflexibility in timing. The Step Up App can be downloaded and installed from the Google Play Store onto mobile devices [11].

The Step Up App is able to dismiss the problems the club faced through its easily accessibility from being downloaded on the smart phone used commonly by high school students. The app itself is not only easy to download and install on smart devices but also has easy to use elements of signing up and logging in and each user having a home screen that contains the various features they may use. These features include but are not limited to "Questions to Me," "Ask Questions," and "Leader Board." The "Ask Questions" tab leads to a large chat room that includes all members. This is a new idea implemented in the club which originally only targeted one on one tutoring. This large real-time chat room allows all students to quickly receive an answer for a question because all members are present in it to answer questions. The leader board was one of the main ideas behind the creation of the app in encouraging more motivation of members. It creates a platform in which all students are able to rate each other in the tutoring reliability and accuracy. This creates a much more trustworthy app that does not permit students from randomly answering others questions without actual knowledge. The leader board also creates an incentive that encourages students to answer others questions and become recognized for doing so.

In the future, there is still much to improve and update for the Step Up App. The Step Up App is still in the processes of developing a more modern styled user interface. The current user face includes each of the features and easy to access. However, a more modern styled user interface could please users in a more aesthetic manner. Additionally, the Step Up App is working on a possible limitation of users exploiting the rating system. Users may purposely vote positively for people they know to allow them to rank on the leader board. Step Up's plan in trying to solve such a scenario is in the making of creating a monitoring system within the board of Step Up. Additionally, Step Up has in plan of trying a one month system of the leader board, which means for every one month, the rating will be cleared and all begin from zero again.

This form of App type that offers a peer-to-peer tutoring is on-demand and has not been largely invested yet but could become an extremely useful application not only within the high school but in all areas and levels of academics.

\section{REFERENCES}

[1] Goodlad, Sinclair, and Beverley Hirst. Peer Tutoring. A Guide to Learning by Teaching. Nichols Publishing, PO Box 96, New York, NY 10024, 1989.

[2] Topping, Keith J. "The effectiveness of peer tutoring in further and higher education: A typology and review of the literature." Higher education 32.3 (1996): 321-345.

[3] Cohen, Jiska. "Theoretical considerations of peer tutoring." Psychology in the Schools 23.2 (1986): 175-186.

[4] Boyer, Ernest L. High school: A report on secondary education in America. Harper \& Row, Inc., 10 East 53rd Street, New York, NY 10022, 1983. 
[5] Fuchs, Lynn S., Douglas Fuchs, and Sarah Kazdan. "Effects of peer-assisted learning strategies on high school students with serious reading problems." Remedial and Special Education 20.5 (1999): 309-318.

[6] "Teens' Ownership of Smartphones Has Surged - eMarketer", Emarketer.com, 2016. [Online]. Available:https://www.emarketer.com/Article/Teens-Ownership-of-Smartphones-Has-Surged/ 1014161. [Accessed: 01- Aug- 2017].

[7] Link, Georg JP, et al. "Evaluating anchored discussion to foster creativity in online collaboration." CYTED-RITOS International Workshop on Groupware. Springer, Cham, 2015.

[8] Moreno, Ismael Solis, et al. "An approach for characterizing workloads in google cloud to derive realistic resource utilization models." Service Oriented System Engineering (SOSE), 2013 IEEE 7th International Symposium on. IEEE, 2013.

[9] J. Bolkan, "Sesh Mobile App Offers Peer-to-Peer Tutoring -- Campus Technology", Campus Technology, 2015. [Online]. Available: https://campustechnology.com/articles/2015/02/04/seshmobile-app-offers-peer-to-peer-tutoring.aspx. [Accessed: 07- Aug- 2017].

[10] "Home - Brainfuse Online Tutoring", Brainfuse Online Tutoring, 2017. [Online]. Available: http://home.brainfuse.com/. [Accessed: 06- Aug- 2017].

[11] “Step Up - Android Apps on Google Play.” Google Play, Google, play.google.com/store/apps/details?id=appinventor.ai_MeghanHaoWang.Tutor. 